\title{
Multi-feature Vegetable Recognition using Machine Learning Approach on Leaf Images
}

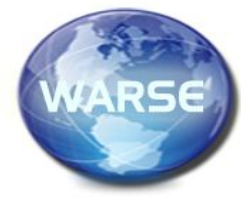

\author{
Nurul Amelina Nasharuddin, Nur Syamimie Mohd Yusoff, Siti Khadijah Ali \\ Department of Multimedia, Faculty Computer Science and Information Technology, \\ Universiti Putra Malaysia, Malaysia \\ \{nurulamelina, ctkhadijah\}@upm.edu.my
}

\begin{abstract}
Vegetables are one of the staple foods that are being consumed daily by Malaysians. With the abundance of the vegetables' type, there are a lot of lookalike vegetables which are from the same species but different type. One of the ways to distinguish the types is by looking at the leaves which are the most visible part of a vegetable. An automated vegetable recognition approach using the colour and shape features of the leaf images is being studied in this work. We focus on the vegetables that mostly consumed by Malaysian. The presented approach was tested on 300 leaf images from six different types of vegetables. Few machine learning classification techniques have been compared, and it was shown that Support Vector Machine technique is the best classifier in this work. The experiments showed that the vegetables can be recognised accurately, up to $95.7 \%$ using the Support Vector Machine when using both features were used. The study revealed that the proposed recognition approach can provide a reliable and faster way to automatically classify vegetables which are common in Malaysia.
\end{abstract}

Key words : colour descriptor, Malaysian vegetable recognition, shape descriptor, support vector machine.

\section{INTRODUCTION}

Locally grown vegetables are available year-round as Malaysia is a tropical country. With the abundance of vegetables, there are a lot of lookalike vegetables that are available in the market. Usually, these vegetables are similar in species but different in type. For example, to differentiate between coriander and celery by using naked eyes is a bit difficult as they are quite the same in size, shape or colour. In the agricultural industry, vegetables are usually being inspected visually by a trained individual for quality assessment. This requires a continual and consistent effort to ensure consistency. Thus, an automatic plant recognition, especially on the vegetables, is useful since it is helpful to classify plants faster and can be implemented in agricultural and industrial tasks [1]. Also with the abundance of high-resolution images, they can be used to facilitate in a lot of research and work such as recognition, classification or information retrieval $[2,3]$.
Before we start the experimentation, we did a survey which was being distributed among Malaysians aged between 18 to 27 years old to test their knowledge in recognising some of the common vegetables. In Malaysia, the total number of the respondents are 20 and from various races. The images of the six types of vegetables that are found to have a similar appearance are being shown to them and they need to classify them according to their types. The types include red spinach (or bayam merah in Malay language), green spinach (bayam hijau), Chinese mustard (sawi Jepun), green mustard (sawi hijau), coriander (daun ketumbar) and celery (daun sup).

The results showed that majority of the respondents, have fair knowledge in recognising the vegetables and there are vegetables that they did not recognise. All of these vegetables will be chosen as the dataset for our proposed recognition approach. The leaf is the most visible part of a vegetable and it can be used to distinguish between the types of vegetables. Other than the leaves, part of a plant that can be use recognition or classification are fruits, flowers and branching styles. For this proposed vegetable recognition approach, the best feature will be the leaf as all the vegetables in the dataset mainly comprises of leaves.

The respondents were also being asked on what features or characteristics of the vegetables that help them most in identifying the types of vegetables. Majority of the respondents $(70 \%)$ chose the shape and colour as the best features. Therefore, we selected both features to test whether the computer's ability is on a par with the human in recognising the vegetable images. The shape is the outer boundary of the leaf and can be differentiated by looking at its area and perimeter. The colour of the vegetable can be differentiated by looking at the darkness of the green colour or the combination of colours on the leaf. We hoped that this proposed work can help the Malaysians to recognise vegetables automatically. It may also help children and visually impaired people in recognising the vegetables.

Therefore, this present work aims to propose an automatic vegetable recognition approach using the leaf's shape and colour features. The six types of common vegetables in 
Malaysia as being listed before that are found to have a similar appearance will being used as the experiment dataset. The rest of this paper is organised as follows. Section 2 discusses some recent related work on plant or leaf recognition. Section 3 and 4 introduce the experimental setup. Results and discussions are discussed in Section 5 along with some suggestions for future work. Section 6 concludes this paper.

\section{RELATED WORKS}

The high demand for agricultural products makes the use of technology in the industry becoming more important [4]. This includes recognising and classifying the type of plant. Classification of the plant by using technology could help a lot in agriculture. It will make the process of separating the various type of agriculture product become fast and accurate. Besides that, by using technology in the classifying process, it will help to ease the agriculture people in handling their work. Vegetable recognition by visual may be easy for individuals who deal with them every day but not for a computer. There are numbers of research have been made on the topic of plant and vegetable classification [5]-[10].

Reference [5] developed an automatic fruit classification technique by fusing three basic features of colour, shape and texture to characterise the objects. There is a total of nine features from the three categories. The combined features of the image scored better although the number of training data is less. They experimented with numbers of classifiers and K-Nearest Neighbour (KNN) gave $97.5 \%$ of accuracy. The also tested the performance of the classifiers and it showed that KNN is not time-consuming as other classifiers plus the result is satisfactory. The authors concluded that single feature descriptor is not enough to capture the characteristics of a class in classification problem. Hence a combination of features might be necessary in order to capture the perfect representation of the image.

Other than the fruit image, a computer-based plant recognition can also use other characteristics of a plant such as leaves, flowers, branching styles, and outlooks. The easier and accurate way is to use leaves to identify the image. There are various leaf features or characteristics that could help in identifying the vegetable type, for example, their shape, size, venation, colour and texture [6,7]. A much more recent study used the shape, image intensities (or statistical features) and venation features [6]. They proposed an algorithm that includes pre-processing, segmentation, feature extraction, dimensionality reduction and classification. Other than handcrafted feature-based extraction approach, they also experimented with a Convolutional Neural Network (CNN) approach of AlexNet. They demonstrated that the AlexNet is feasible to be implemented when the training data is large.

Reference [7] also employed a deep learning method using Probabilistic Neural Network (PNN) to classify leaf images using 12 morphological shape features. They used the
Principal Component Analysis (PCA) for dimensionality reduction of the feature vector before adopting the PNN to do the classification. The average accuracy of their proposed method is $90 \%$ and it is good considering that the proposed framework was easy to be implemented and the processing speed is fast. To get this accuracy, they trained 1800 images of leaves thus this is not a practical approach to our proposed work. We decided to handcraft the feature selection to test on our small dataset. Further, the selection of suitable leaf features is important to achieve a good vegetable recognition system with the most accurate recognition rate.

One of the important dataset in leaf recognition is the Flavia Project dataset of leaf images which were developed by the researchers in [7]. There are around 32 types of plants mostly found in China. Many research did their experiments using this dataset and one of the examples in [8]. We are not using Flavia dataset as our research are focusing on Malaysian vegetables. But in the future, in order to test the robustness of our proposed approach, we can try to conduct the experiment by using the Flavia dataset.

There are two most related studies to our proposed method [9, 10]. The authors in reference [9] classified plant using the leaf images based on shape and colour descriptors. They extracted seven elements in shape features which are the length, width, area, perimeter, hull area, hull perimeter and distance map along horizontal and vertical axes. For the colour features, the authors used the colour histogram as the descriptor for extracting the red, green and blue colour channels. They experimented with the implementation of a single feature and it gave them $83.5 \%$ of accuracy when using the shape feature. However, by using both features and KNN classifier, they were able to achieve $87.3 \%$ of classification accuracy. The authors mentioned that the performance might be affected by the dataset that they used where each image contains many leaves.

The authors in [10] mentioned that most of the leaf identification systems are not fully automated and need of extra-human works. Thus, they suggested an automatic leaf recognition to identify plants. They also chose colour and shape descriptors to represent the leaf image. Based on shape features, the authors extracted geometric and morphological features. For the geometric features, they extracted leaf area, perimeter, diameter, length and width. Using the five features, they defined the morphological features such as perimeter ratio diameter, form factor, aspect ratio and many more.

For the colour feature, they proposed colour histogram in their experiment. They extracted the red, green and blue colour values from the leaf images and calculated their means. The experiment showed that using a single feature could not give the expected result because each plant leaf is invariably similar. They achieved a $93.3 \%$ classification accuracy when using a combination of shape and colour features. Therefore, the combination of features is proven to provide higher 
accuracies in recognising the plant. From these observations, we also selected both shape and colour features in order to test whether computers' ability is on a par with a human in recognising the vegetable images.

\section{EXPERIMENTAL SETUP}

\subsection{Hardware and Software Requirement}

We conducted the experiments mentioned in this study on a desktop running on Microsoft Windows 10 Professional operating system, with Intel ( ${ }^{\circ}$ Core TM i5-3470 CPU, 3.20GHz, and $8 \mathrm{~GB}$ Random Access Memory. MATLAB $\mathrm{R} 2018 \mathrm{a}$ is used for feature extraction and image recognition.

\subsection{Dataset Collection}

To the author's knowledge, there are no publicly available Malaysian vegetables' leaf images that can be used. In this work, we manually take the pictures of the vegetable's leaves as the dataset and pre-processed the images before the feature extraction step. The leaf images are captured using digital cameras with white background and there is no restriction on the direction of leaves when being photographed. A total of 300 images have been captured where each type of vegetable has 50 images. Figure 1 shows sample images of each type.

\section{EXPERIMENTATION}

In order to test the recognition approach, we created a prototype system to show the processes on the user's side. There are two phases in the recognition approach which are the training phase and the recognition phase. In the training phase, the classification algorithms will be used to train the system to recognise the six types of vegetables using the obtained shape and colour feature values. Then, the prototype system will predict the type of input vegetable image during the recognition phase. The overall framework of the recognition approach is shown in Figure 2 and the detailed operation of each step is explained in the following subsections.

\subsection{Pre-processing}

The pre-processing step includes resizing the leaf images and removing any noise in the images in order to enhance their quality. To make all images of equal size, all the input images are first transformed to $128 \times 128$ pixels.

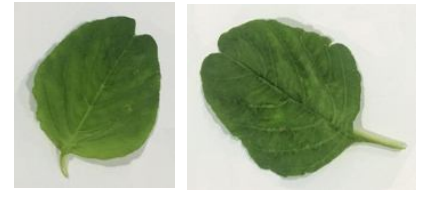

(a)

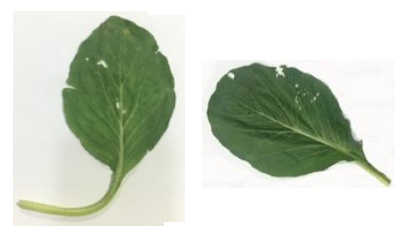

(c)

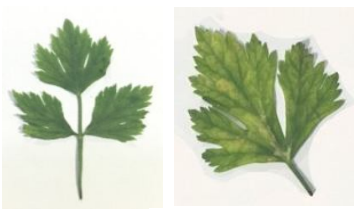

(e)

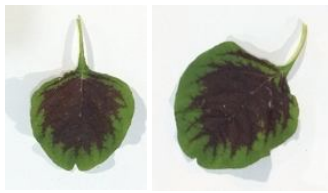

(b)

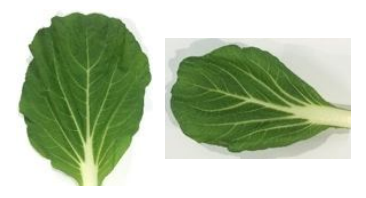

(d)

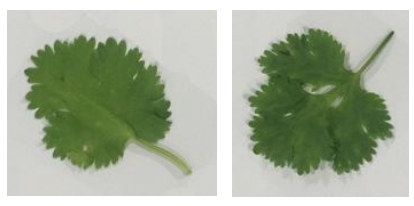

(f)
Figure 1: Sample of vegetable dataset (a) green spinach, (b) red spinach, (c) green mustard, (d) Chinese mustard, (e) celery and (d) coriander

Then the images are converted to a greyscale image of 256 levels. This will make sure the algorithm is less complex compared to using RGB colour space which takes up around three bytes for each pixel of the image. The greyscale images are then are binarized by calculating the threshold using Otsu's algorithm [11]. Values of the pixels that are greater than the threshold are replaced with the 1 (white) and other pixels are replaced with 0 (black). The binarized image is smoothed by filtering noises using a rectangular averaging filter of size $3 \times 3$. Then, the image was convolved using a $3 \times$ 3 Laplacian operator to get the margin of the leaf image.

\subsection{Feature Extraction}

Feature extraction is an essential step to be conducted in any recognition systems. In this vegetable's recognition approach, we chose to extract shape and colour features which are the most basic features for recognition of vegetables. To extract those features from the images, appropriate descriptors need to be selected so that the result produced is accurate.

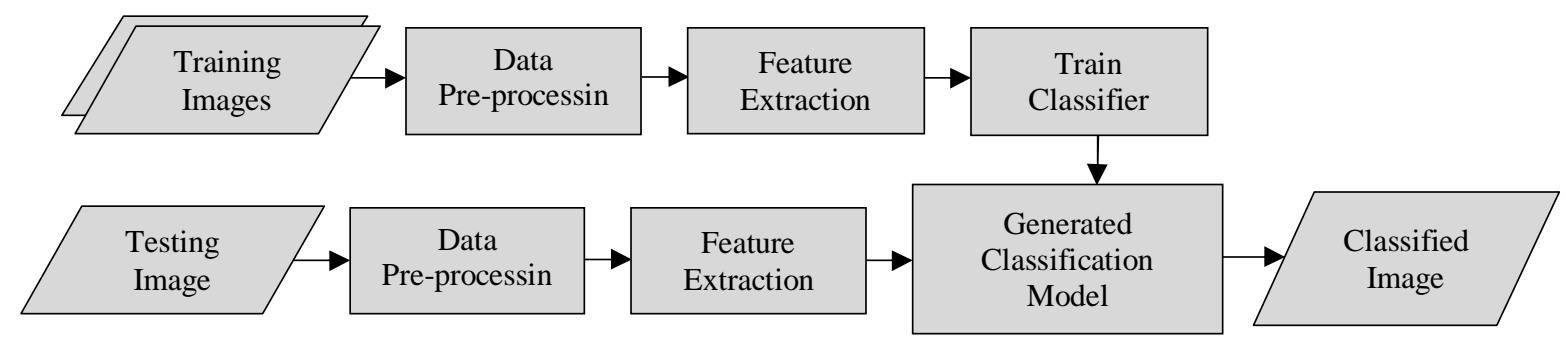

Figure 2: Overall framework on the proposed vegetable recognition system 
In this study, we used two fundamental features which are commonly used in the literature for the shape representation which are the leaf area and the leaf perimeter. The binary leaf image is processed to calculate the smooth factor which measures the difference of areas of two leaves processed. We only need the leaf boundary in order to extract the shape feature. Leaf area gives the total number of pixels for each smoothed leaf and every leaf has its own characteristics. Leaf perimeter calculates the number of pixels which lies on the boundary of processed leaf.

Some of the leaf images in the dataset have very similar shapes and can affect the recognition accuracy. Thus, colour is being chosen as another feature in addition to shape because some of the leaves have different colours. We then extracted colour histogram feature from the leaf images. Colour histogram considers the distribution of colours in an image. The advantage of the method is that the method is not affected by any rotation or translation of the image. A histogram provides a statistical graph that shows the number of pixels of the red, green and blue colours of the image. We used the values of mean $(\mu)$ and standard deviation $(\sigma)$ of intensity values of the red, green and blue channels to form the feature vector based on (1) and (2) below:

$$
\begin{gathered}
\mu=\frac{1}{\mathrm{~N}} \sum_{\mathrm{i}=1}^{\mathrm{N}} \mathrm{x}_{\mathrm{i}} \\
\sigma=\sqrt{\left(\frac{1}{N} \sum_{i=1}^{N}\left(x_{i}-\mu\right)^{2}\right)}
\end{gathered}
$$

where $\mathrm{N}$ is the total number of pixels within an image and $x_{i}$ is the current pixel being processed. Therefore, in total there are four features used as the representation for each image

\subsection{Recognition}

After extracting the shape and colour features from leaf images, we made a preliminary experiment using a few different classification algorithms such as the KNN, Decision Trees, Discriminant Analysis, Support Vector Machines (SVM) and few others in order to recognise the vegetables' types. It was shown that the SVM performed better for this work compared to the other techniques. Thus, we chose SVM to classify the vegetable images in this work. SVM is a type of supervised learning model that is used for a classification task. Each training image will be associated with a class label. In this work, we implemented a multiclass SVM because we have more than two classes that need to be classified. It uses a one-to-one coding design where it will reduce the classification problem to a set of binary classifiers. For example, if there are $n$ classes, then $n(n-1) / 2$ numbers of classifiers will be constructed. The binary classifiers are used to train data between classes at a time. Figure 3(a) shows the process flow of the proposed multi-feature-based extraction while Figure 3(b) visualises the SVM algorithm process.

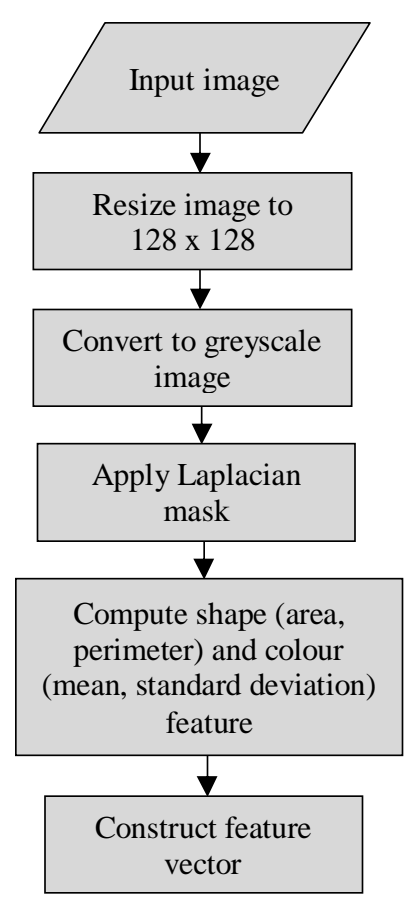

(a)

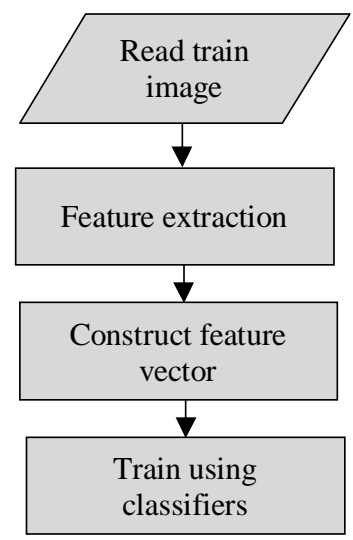

(b)
Figure 3: Process flow for (a) shape and colour feature extraction, . and (b) training of images.

classitied to the class with the nearest distance.

$$
d_{H}(i, j)=\sum_{k=0}^{n=1}\left[y_{i, k} \neq y_{j, k}\right]
$$

$d_{H}$ is the Hamming distance between two objects $i$ and $j, k$ is the index of the respective variable reading $y$ out of the total number of variables $n$. The Hamming distance itself gives the number of mismatches between the variables paired by $k$.

\subsection{Evaluation Measurement}

The comparison between the performance of the algorithm and the truth values were illustrated using a confusion matrix shown in Table 1. True positives and true negatives are the observations that are correctly predicted by the algorithm. A good recognition approach should have a minimal number of false positives and false negatives. The performance of the proposed algorithm was then measured using the accuracy measurement which is the ratio of correctly predicted observation to the total observations, as in (4).

$$
\text { Accuracy }=\frac{T P+T N}{T P+F P+T N+F N}
$$


Table 1: Confusion matrix

\begin{tabular}{|c|c|c|c|}
\hline \multirow{4}{*}{ Actual } & & \multicolumn{2}{|c|}{ Predicted } \\
\hline & & Positive & Negative \\
\hline & Positive & $\begin{array}{l}\text { True Positive } \\
\text { (TP) }\end{array}$ & $\begin{array}{l}\text { False Negative } \\
\text { (FN) }\end{array}$ \\
\hline & Negative & $\begin{array}{l}\text { False Positive } \\
\text { (FP) }\end{array}$ & $\begin{array}{l}\text { True Negative } \\
\text { (TN) }\end{array}$ \\
\hline
\end{tabular}

\section{RESULTS AND DISCUSSION}

The proposed vegetable recognition system was tested on the manually constructed dataset containing leaf images of six vegetables types with 300 samples. We calculated the accuracy of the system using number of correctly recognised vegetables from total images in the dataset.

Three experiments were conducted in this study. For the first experiment, the proposed multi-feature descriptors were evaluated using 300 vegetables images using the top-2 classification algorithms tested in the previous preliminary experiment which are SVM and KNN. In the second experiment, we tested the accuracy values of a cross-validation for ten different folders in order to test the accuracy in a random order. The third experiment calculated the recognition accuracy for each types of the vegetable.

Firstly, we extracted only the shape feature to be used in the recognition. Then, we tested using only the colour feature before we finally combined both features as the feature set in the training phase. Table 2 shows the classification accuracy of single and combination features using top- 2 classification algorithms which are SVM and KNN with k equals to 10 .

Table 2: Accuracy value for single and combined features based on SVM and KNN classifiers

\begin{tabular}{|l|c|c|}
\hline Features & SVM (\%) & KNN (\%) \\
\hline Shape & 77.7 & 82.7 \\
\hline Colour & 89.3 & 91.7 \\
\hline Shape and Colour & $\mathbf{9 5 . 7}$ & $\mathbf{9 3 . 8}$ \\
\hline
\end{tabular}

Based on the result, it showed that by using both shape and colour gives the highest accuracy result or both SVM and KNN. By using the shape feature, SVM achieved $77.7 \%$ of accuracy while $\mathrm{KNN}$ accuracy was better at $82.7 \%$. By using the colour feature, SVM achieved $89.3 \%$ of accuracy and KNN achieved $91.7 \%$ of accuracy. With the combination of shape and colour features, SVM and KNN achieved the highest percentage of accuracy of $95.7 \%$ and $93.8 \%$, respectively. Thus, SVM is proven to be the best classifier for this work which also agreeable by the previous study [12]-[16].

We have also applied 10-fold cross-validation where from each class, $80 \%$ of all the image dataset is used as the training set and the rest as test samples. Table 3 shows a summary of the individual accuracy value for the 10 folders.

Table 3: Accuracy value for cross-validation for 10 folders

\begin{tabular}{|r|c|}
\hline \multicolumn{1}{|c|}{ Folder } & Accuracy (\%) \\
\hline 1 & 93.7 \\
\hline 2 & 95 \\
\hline 3 & 94.7 \\
\hline 4 & 95 \\
\hline 5 & 95 \\
\hline 6 & 95 \\
\hline 7 & 94.3 \\
\hline 8 & 95.3 \\
\hline 9 & 95.3 \\
\hline 10 & 95 \\
\hline Average & $\mathbf{9 4 . 8}$ \\
\hline
\end{tabular}

Based on Table 3, the recognition system using the proposed feature extraction method can achieve higher accuracies in every cross-validation. This result shows that the proposed feature extraction method is effective when it is used on the vegetable dataset. The percentage of the correct classification from each folder sums up to an average of $94.8 \%$ classification accuracy. Table 4 shows the accuracy percentage for each vegetable type. As stated before, all the vegetables chosen as the dataset are based on the appearance of the vegetables which are similar. As we can see in Table 4, the proposed algorithm can successfully recognise all the six types of vegetables.

Table 4: Accuracy for each type of the vegetable

\begin{tabular}{|c|c|}
\hline Types & Accuracy (\%) \\
\hline Green spinach (Bayam Hijau) & 87.6 \\
\hline Red spinach (Bayam Merah) & 88.7 \\
\hline Chinese mustard (Sawi Jepun) & 88.6 \\
\hline Green mustard (Sawi Hijau) & 88.2 \\
\hline Coriander (Daun Ketumbar) & 87.6 \\
\hline Celery (Daun Sup) & 85.9 \\
\hline Average & 87.8 \\
\hline
\end{tabular}

\section{CONCLUSION}

This study is just a preliminary study on creating the dataset for Malaysian vegetables and used them in an automated recognition system. There are a few limitations of this current work that can further be improved. One of them is the proposed approach need to be benchmarked with other methods such as in [4] and [16] in order to justify whether the approach is on par with the current recognition approaches or not.

More feature descriptors can also be extracted from the images as the shape and colour are the most basic features and there are a lot of other features that are suitable and can be used to recognise leaf images. Examples of the features are 
size, texture and venation pattern. The more features that can be extracted from the vegetables' images, the more detailed representation of the images we can achieve. Thus, the accuracy of the proposed method can be increased.

A good system that can classify the type of vegetables automatically could help people a lot in doing their agriculture works. They can save their energy, time and effort in finding information regarding the vegetables. Therefore, this proposed vegetable classification system can provide a reliable and faster way to automatically classify the vegetables.

For future work, we plan to expand the dataset by including more images for each vegetable category as well as exploring other plant categories. More feature descriptors will also be included with a comparison with other recognition techniques. A mobile-based application will also be considered to make it easier for general users or botanist to utilise the recognition application in daily work.

\section{ACKNOWLEDGEMENT}

Authors thanks A. A. Halin and A. H. Jantan for advises and insights throughout the development of this work.

\section{REFERENCES}

1. J. X. Du, X. Wang, and G. -J. Zhang. Leaf shape based plant species recognition, Appl. Mathematics Comput., Vol. 185, pp. 883-893, 2007. https://doi.org/10.1016/j.amc.2006.07.072

2. B. Buvaneswari and T. Kalpalatha Reddy. ELSA-A Novel Technique to Predict Parkinson's Disease in Bio-Facial Recognition System, Int. J. Adv. Trends Comput. Sci. Eng., Vol. 8, pp. 12-17, 2019. https://doi.org/10.30534/ijatcse/2019/03812019

3. D. Sarala, T. Kanikdaley, S. Jogi and R. K. Chaurasiya. Content-Based Image Retrieval Using Hierarchical Color and Texture Similarity Calculation, Int. J. $A d v$. Trends Comput. Sci. Eng., Vol. 7, pp. 11-16, 2018. https://doi.org/10.30534/ijatcse/2018/02722018

4. K. Hameed, D. Chai and A. Rassau. A comprehensive review of fruit and vegetable classification techniques, Image Vision Comput., Vol. 80, pp. 24-44, 2018. https://doi.org/10.1016/j.imavis.2018.09.016

5. L. Rajasekar and D. Sharmila. Performance analysis of soft computing techniques for the automatic classification of fruit dataset, Soft Comput., Vol. 23, pp. 2773-2788, 2019. https://doi.org/10.1007/s00500-019-03776-Z

6. G. Saleem, M. Akhtar, N. Ahmed and W. S. Qureshi. Automated analysis of visual leaf shape features for plant classification, Comput. Electr. Agriculture, Vol. 157, pp. 270-280, 2019. https://doi.org/10.1016/j.compag.2018.12.038

7. S. G. Wu, F. S. Bao, E. Y. Xu, Y. -X. Wang, Y. -F. Chang and Q. -L. Xiang. A leaf recognition algorithm for plant classification using probabilistic neural network, in Proc. IEEE $7^{\text {th }}$ Int. Symp. Signal Process. Inf. Technol., Egypt, 2007, pp. 1-6.

8. A. Caglayan, O. Guclu and A. B. Can. A plant recognition approach using shape and color features in leaf images, in Image Analysis Process. A. Petrosino, Ed. Berlin Heidelberg: Springer, 2013, pp. 161-170. https://doi.org/10.1007/978-3-642-41184-7_17

9. T. Munisami, M. Ramsurn. S. Kishnah, and S. Pudaruth, Plant leaf recognition using shape features and colour histogram with k-nearest neighbour classifier, Procedia Comput. Sci., Vol. 58, pp. 740-747. https://doi.org/10.1016/j.procs.2015.08.095

10. V. Satti, A. Satya, and S. Sharma. An automatic leaf recognition system for plant identification using machine vision technology, Int. J. Eng. Sci. Tech., Vol. 5, pp. 874-879, 2013.

11. N. Otsu. A threshold selection method from gray-level histograms, IEEE Trans. Sys. Man., Vol. 9, pp. 62-66, 1979. https://doi.org/10.1109/TSMC.1979.4310076

12. J. Waldchen and P. Mader. Plant species identification using computer vision techniques: a systematic literature review, Arch. Compu. Methods Eng., Vol. 25, pp. 507-543, 2018.

13. K. Pankaja and G. Thippeswamy. Survey on leaf recognition and classification, in Proc. Int. Conf. Innovative Mechanisms Industry Appl., Bangalore, 2017, pp. 443-450.

14. Z. Ibrahim, N. Sabri and N. N. Abu Mangshor. Leaf recognition using texture features for herbal plant identification, Indonesian J. Electr. Eng. Comput. Sci., Vol. 9, pp. 152-156, 2018. https://doi.org/10.11591/ijeecs.v9.i1.pp152-156

15. M. Lukic, E. Tuba and M. Tuba. Leaf recognition algorithm using support vector machine with hu moments and local binary patterns, in Proc. IEEE $15^{\text {th }}$ Int. Symp. Appl. Mach. Intell. Inf., Slovakia, 2017, pp. 485-490.

16. H. X. Kan, L. Jin and F. L. Zhou. Classification of medical plant leaf image based on multi-feature extraction, Pattern Recog. Image Anal., Vol. 27, pp. 581-587, 2017.

https://doi.org/10.1134/S105466181703018X 